Pacific Journal of Mathematic 


\title{
PRIMARY POWERS OF A PRIME IDEAL
}

\author{
L. RobBiano AND G. VALla
}

\begin{abstract}
In this paper we show that the powers of a prime ideal $\mathfrak{p}$ are primary iff the direct summands of the graded ring associated with $\mathfrak{p}$ are torsion-free. We prove some consequences of this fact especially in connection with geometric situations.
\end{abstract}

Let $k$ be a field, $Y, X$ closed subschemes of $\boldsymbol{P}_{k}^{n}$; suppose that $Y$ is irreducible, reduced and contained in $X$ and let $\mathfrak{p}$ be the prime ideal corresponding to $Y$ in the homogeneous coordinate ring of $X$ (which is the unique ring $B=k\left[x_{0}, \cdots, x_{n}\right]$ such that $X=\operatorname{Proj}(B)$ and $\left(x_{0}, \cdots, x_{n}\right)$ does not belong to Ass $\left.(B)\right)$. Is it true that $\mathfrak{p}^{n}$ is primary for every $n$ ?

The general answer is of course in the negative (see for instance Corollary 3.2). On the other hand it is well known that the answer is in the affirmative if $k=\boldsymbol{C}, X=\boldsymbol{P}_{k}^{n}$ and $Y$ is a complete intersection; this fact has been improved by Bonardi in [1] and recently by Hochster, who more generally proved that $\mathfrak{p}^{n}$ is primary for all $n$ if $\mathfrak{p}$ is a prime ideal generated by a regular sequence in a domain (see [3]).

The main purpose of this paper is the study of the case where $Y$ is not a complete intersection in $X$, and we get two essentially different situations when $\operatorname{dim}(Y)=0$ and $\operatorname{dim}(Y)>0$. More precisely, if $Y$ is a closed rational point, we get the following complete answer: $\mathfrak{p}^{n}$ is primary for all $n$ if and only if $x$ is a "cone" having $Y$ in its vertex (for precise statement see Theorem 3.1). Instead, when $\operatorname{dim}(Y)>0$, if $Y$ and $X$ are complete intersections in $\boldsymbol{P}_{k}^{n}$, such that $Y$ is nonsigular and $X$ is nonsingular in the points of $Y$, then $\mathfrak{p}^{2}$ is primary and $\mathfrak{p}^{n}$ is primary for all $n$ if we add the condition $\operatorname{dim}(Y) \geqq \operatorname{codim}(X)$ (see Theorem 3.3), in particular if $X$ is a hypersurface (Corollary 3.4).

Suitable example at the end of $\S 3$ justify the hypotheses we need in the above mentioned theorems.

As to the proofs, first we develop criteria for $\mathfrak{p}^{n}$ to be primary, showing that this property is connected with the fact that certain modules are torsion-free (Proposition 1.1 and Corollaries). Then, essentially using homological methods, we can prove "algebraic" theorems (Theorems 2.2 and 2.3), from which the "geometric" ones easily follow.

In this paper all rings are supposed to be commutative, noetherian and with identity. 
1. Let $B$ be a ring, $\mathfrak{p}$ a prime ideal and $A=B / \mathfrak{p}$; denote by $G_{n}$ the $A$-module $\mathfrak{p}^{n} / \mathfrak{p}^{n+1}$ and by $G(\mathfrak{p})$ the graded $A$-algebra $\bigoplus_{n=0}^{\infty} G_{n}$ (for more details see [6]).

Proposition 1.1. If $N$ is a positive integer, the following conditions are equivalent:

(a) $\mathfrak{p}^{n}$ is primary for $n \leqq N$.

(b) $G_{n}$ is torsion-free for $n \leqq N-1$.

Proof. It is clear that $\mathfrak{p}^{n}$ primary implies $G_{n-1}$ torsion-free. Let $n \leqq N, x \notin \mathfrak{p}, y \notin \mathfrak{p}^{n}$. If $y \in \mathfrak{p}^{r}, y \notin \mathfrak{p}^{r+1}$ then $0 \leqq r<n$; we have $0 \neq$ $\bar{x} \in A, 0 \neq \bar{y} \in G_{r}$, therefore $\bar{x} \bar{y} \neq 0$ or, which is the same, $x y \notin \mathfrak{p}^{r+1}$; but we have $\mathfrak{p}^{r+1} \supseteqq \mathfrak{p}^{n}$ and so $x y \notin \mathfrak{p}^{n}$.

REMARK 1. As a consequence of Proposition 1.2 we get that $\mathfrak{p}^{2}$ is primary iff $G_{1}$ is torsion-free. Nevertheless the following example shows that if $n>2 \mathfrak{p}^{n}$ need not to be primary even if $G_{n-1}$ is torsionfree. Let $B=k[x, y, z] \cong k[X, Y, Z] /\left(Y^{2}, Y Z, X Y-Z^{3}\right)$ and $\mathfrak{p}=(y, z)$. We get in this case $G(\mathfrak{p}) \cong k[X]\left[T_{1}, T_{2}\right] /\left(X T_{1}, T_{1} T_{2}, T_{1}^{2}, T_{2}^{4}\right)$, hence $G_{2}$ is torsion-free, but $\mathfrak{p}^{3}$ is not primary.

REMARK 2. In the above example $\mathfrak{p}^{4}$ is primary and this shows that $G_{N}$ torsion-free does not imply $G_{n}$ torsion-free for $n<N$.

COROLlaRY 1.2. The following conditions are equivalent:

(a) $\mathfrak{p}^{n}$ is primary for every $n$.

(b) $G(\mathfrak{p})$ is torsion-free.

(c) The canonical homomorphism $G(\mathfrak{p}) \rightarrow G\left(\mathfrak{p} B_{\mathfrak{p}}\right)$ is injective.

In particular $G(\mathfrak{p})$ is a domain iff $\mathfrak{p}^{n}$ is primary for every $n$ and $G\left(\mathfrak{p} B_{\mathfrak{p}}\right)$ is a domain.

Proof. It follows from Proposition 1.1 that (a) and (b) are equivalent. Denoting with $K$ the quotient field of $A$, the equivalence of (b) and (c) easily follows after remarking that $G\left(\mathfrak{p} B_{\mathfrak{p}}\right) \cong G(\mathfrak{p}) \bigotimes_{A} K$.

COROLlaRY 1.3. If $\mathfrak{p}$ is locally generated by a regular sequence, $\mathfrak{p}^{n}$ is primary for all $n$. In particular if $V(\mathfrak{p})$ is regular in $\operatorname{Spec}(B)$ and $\operatorname{Spec}(A)$ is regular, $\mathfrak{p}^{n}$ is primary for every $n$.

Proof. For an ideal it is clear that to be primary is a local property, hence we may assume that $\mathfrak{p}$ is generated by a regular sequence and the conclusion follows since $G(\mathfrak{p})$ is a polynomial ring over $A$ (see [5] Theorem 2.1). 
2. Let $B$ be a ring, $\mathfrak{p}$ a prime ideal and $A=B / \mathfrak{p}$.

LEMMA 2.1. The following conditions are equivalent:

(a) $\mathfrak{p}$ is locally generated by a regular sequence.

(b) $\mathfrak{p} / \mathfrak{p}^{2}$ is a projective A-module and $B_{\Downarrow}$ is regular.

(c) $\mathfrak{p}^{n} / \mathfrak{p}^{n+1}$ is a projective $A$-module for all $n$ and $B_{\mathfrak{p}}$ is regular.

Proof. We may assume that $B$ is local. If $\mathfrak{p}$ is generated by a regular $B$-sequence, $B_{\mathfrak{p}}$ is regular and since $G(\mathfrak{p})$ is a polynomial ring over $A, \mathfrak{p}^{n} / \mathfrak{p}^{n+1}$ is a free $A$-module for all $n$. Let us now assume that (b) holds, and $\bar{a}_{1}, \cdots, \bar{a}_{r} \in \mathfrak{p} / \mathfrak{p}^{2}$ be a free basis over $A$. Using Nakayama we get $\mathfrak{p}=\left(a_{1}, \cdots, a_{r}\right)$, hence $\mathfrak{p} B_{\mathfrak{p}}=\left(a_{1} \cdots, a_{r}\right) B_{\mathfrak{p}}$; we claim that $\left(a_{1}, \cdots, a_{r}\right)$ is a minimal basis for $\mathfrak{p} B_{p}$. On the contrary, let $\mathfrak{p} B_{\mathfrak{p}}$ be generated by a proper subset of $\left\{a_{1}, \cdots, a_{r}\right\}$ say $\left\{a_{2}, \cdots, a_{r}\right\}$; then there exists an element $t$ not in $\mathfrak{p}$ such that $t a_{1} \in\left(a_{2}, \cdots, a_{r}\right) B$, which contradicts the hypothesis that $\bar{a}_{1}, \cdots, \bar{a}_{r}$ are linearly independent over $A$. Combining with $B_{\mathfrak{p}}$ regular, it follows that $a_{1}, \cdots, a_{r}$ is a regular $B_{\mathrm{p}}$-sequence. Let us now consider the graded homomorphism $\varphi: A\left[T_{1}, \cdots, T_{r}\right] \rightarrow G(\mathfrak{p})$ defined by $\varphi\left(T_{i}\right)=\bar{a}_{i} \in \mathfrak{p} / \mathfrak{p}^{2}$. It is clear that $\phi$ is onto, hence $G(\mathfrak{p}) \cong A\left[T_{1}, \cdots, T_{r}\right] / I$. On the other hand $G\left(\mathfrak{p} B_{\mathfrak{p}}\right) \cong G(\mathfrak{p}) \bigotimes_{A} K \cong K\left[T_{1}, \cdots, T_{r}\right](K=$ quotient field of $A)$ because $a_{1}, \cdots, a_{r}$ is a regular $B_{p}$-sequence generating $\mathfrak{p} B_{p}$. It follows that $I \otimes_{A} K=0$, hence $I=0$ as $I$ is obviously a torsion-free $A$-module. Applying Rees criterion (see [5] Theorem 2.2) we get that $a_{1}, \cdots, a_{r}$ is a regular $B$-sequence.

THEOREM 2.2. If $B_{\mathfrak{p}}$ and $A$ are regular and $\operatorname{dim}(A)=1$ the following conditions are equivalent:

(a) $\mathfrak{p}^{2}$ is primary.

(b) $\mathfrak{p}^{n}$ is primary for every $n$.

(c) $V(\mathfrak{p})$ is regular in $\operatorname{Spec}(B)$.

(d) $\mathfrak{p}$ is locally generated by a regular sequence.

(e) $\mathfrak{p} / \mathfrak{p}^{2}$ is a projective A-module.

(f) $\mathfrak{p}^{n} / \mathfrak{p}^{n+1}$ is a projective A-module for every $n$.

Proof. It is obvious that (b) implies (a). By Lemma 2.1 (d), (e), (f) are equivalent and (d) implies (b) by Corollary 1.3. If now $\mathfrak{p}^{2}$ is primary, by Proposition $1.1 \mathfrak{p} / \mathfrak{p}^{2}$ is a finitely generated torsionfree $A$-module, hence projective, because $A$ is a Dedekind domain and so (a) implies (e). The equivalence between (c) and (d) is well known since $A$ is regular.

Lemma 2.3. Let $A$ be a domain, $M$ a finitely generated $A$-module, and a an ideal of $A$ such that h.d. ${ }_{A} M<\operatorname{gr}(\mathfrak{a})$ and $M_{\mathrm{p}}$ is torsion- 
free for every prime $\mathfrak{p}$ such that $\mathfrak{a} \not \subset \mathfrak{p}$. Then $M$ is torsion-free.

Proof. If not, we can choose a prime $\mathfrak{p} \neq(0)$ such that $\mathfrak{p} \in \operatorname{Ass}(M)$, and then $\mathfrak{p} A_{\mathfrak{p}} \in \operatorname{Ass}\left(M_{\mathfrak{p}}\right)$, hence depth $\left(M_{\mathfrak{p}}\right)=0$. Therefore h.d. $\cdot_{\mathfrak{p}} M_{\mathfrak{p}}=$ $\operatorname{gr}\left(\mathfrak{p} A_{\mathfrak{p}}\right)$. But h.d. $\cdot_{A_{\mathfrak{p}}}\left(M_{\mathfrak{p}}\right) \leqq \mathrm{h} . \mathrm{d}_{\cdot_{A}} M$ and so $\operatorname{gr}\left(\mathfrak{p} A_{\mathfrak{p}}\right) \leqq \mathrm{h} . \mathrm{d}_{\cdot_{A}} M<\operatorname{gr}(\mathfrak{a})$; this implies $\mathfrak{a} \not \subset \mathfrak{p}$, then $M_{\mathfrak{p}}$ is torsion-free over $A_{\mathfrak{p}}$, a contradiction.

Theorem 2.4. Let $R$ be a ring, a, $\mathfrak{P}, \mathfrak{n}$ ideals such that $\mathfrak{a}, \mathfrak{P}$ are locally generated by regular sequence, $\mathfrak{P}$ is prime and $\mathfrak{a} \subseteq \mathfrak{P} \subseteq \mathfrak{n}$. If $V(\mathfrak{P} / \mathfrak{a})-V(\mathfrak{n} / \mathfrak{a})$ is regular in $\operatorname{Spec}(R / \mathfrak{a})$, Spec $(R / \mathfrak{P})-V(\mathfrak{n} / \mathfrak{P})$ is regular and $\operatorname{gr}(\mathfrak{n} / \mathfrak{P})=d \geqq 2$, we have:

(a) $(\mathfrak{P} / \mathfrak{a})^{2}$ is primary

(b) If $d>\operatorname{gr}\left(\mathfrak{a} R_{\mathrm{m}}\right)$ for every maximal ideal $\mathfrak{m} \supseteq \mathfrak{t}$ (for istance if $d>\operatorname{dim} R)$, then $(\mathfrak{P} / \mathfrak{a})^{n}$ is primary for every $n$.

Proof. Using Corollary 1.3 and the local character of the property of being primary, we can restrict our attention to the maximal ideals containing $\mathfrak{n}$. Hence we may assume that $R$ is local.

We shall denote by $a_{1}, \cdots, a_{r}$ the elements of the regular $R$ sequence generating $a$, by $a_{i}$ the ideal $\left(a_{1}, \cdots, a_{i}\right)\left(a_{0}=0\right)$, by $B$ the ring $R / \mathfrak{a}$, by $B_{i}$ the ring $R / \mathfrak{a}_{i}$, by $\mathfrak{p}$ the ideal $\mathfrak{P} / \mathfrak{a}$ in $B$ and by $A$ the ring $R / \mathfrak{P} \cong B / \mathfrak{p}$.

We shall give the proof in several steps.

1. $V\left(\mathfrak{P} / \mathfrak{a}_{i}\right)-V\left(\mathfrak{n} / \mathfrak{a}_{i}\right)$ is regular in $\operatorname{Spec}\left(B_{i}\right)$ for $i=1, \cdots, r$. It follows from the property that a local ring is regular if its quotient by a regular sequence is regular.

2. $\quad a_{i} \notin \mathfrak{P}^{2}+\left(a_{1}, \cdot, \hat{a}_{i}, \cdot, a_{r}\right)$ for $i=1, \cdots, r$. If we denote by $\mathfrak{a}_{\hat{i}}$ the ideal $\left(a_{1}, \cdot, \hat{a}_{i}, \cdot, a_{r}\right)$, the ring

$$
B_{\mathfrak{p}} \cong R_{\Re} / a R_{\Re} \cong\left(R_{\Re} / a_{\hat{i}} R_{\Re}\right) /\left(\bar{a}_{i}\right)
$$

is regular, hence $a_{i} \notin \mathfrak{P}^{2} R_{\mathfrak{P}}+\mathfrak{a}_{\hat{i}} R_{\mathfrak{\wp}}$.

3. Let $s, N$ be integers $0 \leqq s \leqq r, 0<N$; if $\left(\mathfrak{B} / \mathfrak{a}_{i}\right)^{t}$ is primary for every nonnegative integer $i \leqq s-1$ and for every $t=1, \cdots, N$, then $\mathfrak{a}_{s} \cap \mathfrak{P}^{t}=\mathfrak{a}_{s} \mathfrak{P}^{t-1}$ for every $t=1, \cdots, N$.

The proof is by induction on $s$; the case $s=0$ is trivial. Hence we may assume $s \geqq 1$ and $\mathfrak{a}_{s-1} \cap \mathfrak{P}^{t}=\mathfrak{a}_{s-1} \mathfrak{P}^{t-1}$ for every $t=1, \cdots, N$. Let now $\sum_{i=1}^{s} a_{i} x_{i} \in \mathfrak{S}^{t}$, then $a_{s} x_{s} \in \mathfrak{P}^{t}+\mathfrak{a}_{s-1}$; by step $2 a_{s} \notin \mathfrak{P}^{2}+\mathfrak{a}_{s-1}$ so $0 \neq \bar{a}_{s} \in\left(\mathfrak{P} / \mathfrak{a}_{s-1}\right) /\left(\mathfrak{P} / \mathfrak{a}_{s-1}\right)^{2}$. Let $x_{s} \in \mathfrak{P}^{m}+\mathfrak{a}_{s-1}, \quad x_{s} \notin \mathfrak{P}^{m+1}+\mathfrak{a}_{s-1}$, thus $0 \neq \bar{x}_{s} \in\left(\mathfrak{P} / \mathfrak{a}_{s-1}\right)^{m} /\left(\mathfrak{P} / \mathfrak{a}_{s-1}\right)^{m+1}$. On the other hand, by Proposition 1.1, $\bigoplus_{j=1}^{N}\left(\mathfrak{P} / \mathfrak{a}_{s-1}\right)^{j-1} /\left(\mathfrak{P} / \mathfrak{a}_{s-1}\right)^{j}$ can be imbedded as a graded module in $G\left(\mathfrak{P} / \mathfrak{a}_{s-1}\right) \bigotimes_{A} K$ (where $K$ is the quotient field of $A$ ), which is an 
integral domain by Corollary 1.3 and step 1 . Therefore, if $m<t-1$, $0 \neq \bar{a}_{s} \bar{x}_{s} \in\left(\mathfrak{P} / \mathfrak{a}_{s-1}\right)^{m+1} /\left(\mathfrak{P} / \mathfrak{a}_{s-1}\right)^{m+2}$ i.e. $a_{s} x_{s} \notin \mathfrak{P}^{m+2}+\mathfrak{a}_{s-1}$ hence $a_{s} x_{s} \notin \mathfrak{F}^{t}+$ $\mathfrak{a}_{s-1}$, a contradiction. In conclusion $m \geqq t-1$ and so $x_{s} \in \mathfrak{P}^{t-1}+\mathfrak{a}_{s-1}$. We get $\sum_{i=1}^{s} a_{i} x_{i}=\sum_{i=1}^{s-1} a_{i} y_{i}+a_{s} y_{s}$ with $y_{s} \in \mathfrak{P}^{t-1}$; hence $\sum_{i=1}^{s-1} a_{i} y_{i} \in$ $a_{s-1} \cap \mathfrak{P}^{t}=\mathfrak{a}_{s-1} \mathfrak{P}^{t-1}$ by induction and the conclusion immediately follows.

4. The following sequence of $A$-modules is exact for all $t$ and $i$.

(1) $0 \longrightarrow \mathfrak{a}_{\imath} \cap \mathfrak{P}^{t} / \mathfrak{a}_{i} \cap \mathfrak{P}^{t+1} \longrightarrow \mathfrak{P}^{t} / \mathfrak{P}^{t+1} \longrightarrow \mathfrak{a}_{i}+\mathfrak{S}^{t} / \mathfrak{a}_{i}+\mathfrak{P}^{t+1} \longrightarrow 0$

The proof is standard.

5. $\left(\mathfrak{B} / \mathfrak{a}_{s}\right)^{2}$ is primary for $s \leqq r$. If $s=0$ it follows from Corollary 1.3. Therefore we may assume that $\left(\mathfrak{P} / \mathfrak{a}_{i}\right)^{2}$ is primary for $i \leqq s-1$. Using step 3 we get $\mathfrak{a}_{s} / \mathfrak{a}_{s} \cap \mathfrak{P}^{2} \cong \mathfrak{a}_{s} / \mathfrak{a}_{s} \mathfrak{P}$. This is a free $A$-module generated by $\bar{a}_{1}, \cdots, \bar{a}_{s}$; indeed if $a_{1} x_{1}+\cdots+a_{s} x_{s} \in \mathfrak{a}_{s} \mathfrak{P}$, we get $\sum_{i=1}^{s} a_{\imath} x_{i}=\sum_{i=1}^{s} a_{i} y_{i}$ with $y_{i} \in \mathfrak{P}$, hence $x_{i}-y_{i} \in \mathfrak{a}_{s} \subseteq \mathfrak{P}$. Using the exact sequence (1) with $t=1, i=s$ we get h.d. ${ }_{A}\left(\mathfrak{P} / \mathfrak{a}_{s}\right) /\left(\mathfrak{P} / \mathfrak{a}_{s}\right)^{2} \leqq 1$.

On the other hand by step $1 V\left(\mathfrak{P} / \mathfrak{a}_{s}\right)-V\left(\mathfrak{m} / \mathfrak{a}_{s}\right)$ is regular in $\operatorname{Spec}\left(B_{s}\right)$ and by hypothesis $\operatorname{Spec}(R / \mathfrak{P})-V(\mathfrak{n} / \mathfrak{P})$ is regular; applying Corollary 1.3 we get that $\left(\mathfrak{P} / a_{s}\right)^{2}$ is primary at every point of Spec $\left(B_{s}\right)-V\left(\mathfrak{n} / \mathfrak{a}_{s}\right)$, hence by Proposition $1.1\left(\mathfrak{P} / \mathfrak{a}_{s}\right) /\left(\mathfrak{P} / \mathfrak{a}_{s}\right)^{2}$ is torsionfree at the same points. Applying Lemma 2.3, we are through.

6. Let $s$ be an integer, $1 \leqq s \leqq r$. If $\left(\mathfrak{P} / \mathfrak{a}_{i}\right)^{t}$ is primary for $i=0, \cdots, s-1$ and for every $t$, the following exact sequence of $A$-modules holds for all $t$ :

$$
\begin{aligned}
& 0 \longrightarrow \mathfrak{a}_{s-1} \cap \mathfrak{P}^{t} / \mathfrak{a}_{s-1} \cap \mathfrak{P}^{t+1} \longrightarrow \mathfrak{a}_{s} \cap \mathfrak{P}^{t} / \mathfrak{a}_{s} \cap \mathfrak{P}^{t+1} \\
& \stackrel{\varphi}{\longrightarrow} \mathfrak{a}_{s-1}+\mathfrak{P}^{t-1} / \mathfrak{a}_{s-1}+\mathfrak{P}^{t} \longrightarrow 0
\end{aligned}
$$

The first homomorphism is the canonical one. Let $\bar{a}$ be an element of $\mathfrak{a}_{s} \cap \mathfrak{P}^{t} / \mathfrak{a}_{s} \cap \mathfrak{P}^{t+1}$; by step $3 a=\sum_{i=1}^{s} a_{i} x_{i}, \quad x_{i} \in \mathfrak{P}^{t-1}$. We define $\varphi(\bar{a})=\bar{x}_{s}$ and the exactness easily follows.

7. If $0 \leqq s<d,\left(\mathfrak{P} / \mathfrak{a}_{s}\right)^{t}$ is primary for every $t$. We shall prove by induction on $s$ that h.d. ${ }_{A}\left(\mathfrak{a}_{s} \cap \mathfrak{P}^{t} / \mathfrak{a}_{s} \cap \mathfrak{P}^{t+1}\right) \leqq s-1$ for all $t$, h.d. ${ }_{1}\left(\mathfrak{a}_{s}+\mathfrak{P}^{t} / \mathfrak{a}_{s}+\mathfrak{P}^{t+1}\right) \leqq s$ for all $t$ and $\left(\mathfrak{P} / \mathfrak{a}_{s}\right)^{t}$ is primary for all $t$ (we use the convention that h.d. ${ }_{A} M=-1$ if $M$ is the $A$-module with unique element 0 ).

The case $s=0$ is clear. Letus now suppose that

$$
\text { h.d. }{ }_{A}\left(\mathfrak{a}_{s-1} \cap \mathfrak{P}^{t} / \mathfrak{a}_{s-1} \cap \mathfrak{P}^{t+1}\right) \leqq s-2
$$


for all $t$, h.d. ${ }_{A}\left(\mathfrak{a}_{s-1}+\mathfrak{P}^{t} / \mathfrak{a}_{s-1}+\mathfrak{P}^{t+1}\right) \leqq s-1$ for all $t$ and $\left(\mathfrak{P} / \mathfrak{a}_{i}\right)^{t}$ is primary for $i \leqq s-1$ and for every $t$. By step 4 and 6 , the exact sequences (1) and (2) hold for any $t$ and for $i=s$. From (2) and Theorem B ([4] pg. 124) we get h.d. ${ }_{A}\left(\mathfrak{a}_{s} \cap \mathfrak{P}^{t} / \mathfrak{a}_{s} \cap \mathfrak{P}^{t+1}\right) \leqq$ $\max \left(\right.$ h.d. ${ }_{A}\left(\mathfrak{a}_{s-1} \cap \mathfrak{S}^{t} / \mathfrak{a}_{s-1} \cap \mathfrak{P}^{t+1}\right)$, h.d. $\left.\cdot_{A}\left(\mathfrak{a}_{s-1}+\mathfrak{S}^{t-1} / \mathfrak{a}_{s-1}+\mathfrak{P}^{t}\right)\right)=s-1$ for all $t$, hence we deduce from (1) h.d. ${ }_{A}\left(a_{s}+\mathfrak{P}^{t} / \mathfrak{a}_{s}+\mathfrak{P}^{t+1}\right) \leqq s<d$ for all $t$. Using Lemma 2.3 and the same kind of argument of step 5 we get that $\mathfrak{a}_{s}+\mathfrak{P}^{t} / \mathfrak{a}_{s}+\mathfrak{P}^{t+1}$ is torsion-free for all $t$. Hence, by Proposition 1.1, $\left(\mathfrak{P} / \mathfrak{a}_{s}\right)^{t}$ is primary for all $t$.

8. (Conclusion.) Applying step 5 with $s=r$ we get (a); applying step 7 with $s=r$ we get (b).

3. In this section $k$ will denote a field and $\boldsymbol{P}_{k}^{n}$ the $n$-dimensional projective space over $k$; if $a$ is a homogeneous ideal of $k\left[X_{0}, \cdots, X_{n}\right]$, we shall denote by $V=V(\mathfrak{a})=\operatorname{Proj}\left(k\left[X_{0}, \cdots, X_{n}\right] / \mathfrak{a}\right)$ the associated projective scheme. If $P$ is a closed rational point on $V$ and $\mathfrak{p}$ the homogeneous prime ideal of $k\left[x_{0}, \cdots, x_{n}\right] \cong k\left[X_{0}, \cdots, X_{n}\right] / \mathfrak{a}$ corresponding to $P$, we may assume in the following that $P=(1,0, \cdots, 0)$, hence $\mathfrak{p}=\left(x_{1}, \cdots, x_{n}\right)$.

THEOREM 3.1. With the same assumptions, the following conditions are equivalent:

(a) $\mathfrak{p}^{n}$ is primary for every $n$.

(b) a is generated by forms in $k\left[X_{1}, \cdots, X_{n}\right]$.

Proof. Let $a=\left(F_{1}, \cdots, F_{r}\right), F_{i} \in k\left[X_{1}, \cdots, X_{n}\right]$ and $R=$ $k\left[X_{1}, \cdots, X_{n}\right] / a^{*} \quad$ where $\quad a^{*}=\left(F_{1}, \cdots, F_{r}\right) k\left[X_{1}, \cdots, X_{n}\right]$; we get $k\left[X_{0}, \cdots, X_{n}\right] / \mathfrak{a} \cong R\left[X_{0}\right]$ and so $\mathfrak{p}$ is the extension to $R\left[X_{0}\right]$ of a maximal ideal of $R$. Hence (b) implies (a). Now we prove that (a) implies (b). Let $\mathfrak{a}=\left(F_{1}, \cdots, F_{r}\right)$; we may write $F_{1}=X_{0}^{m-1} G_{1}\left(X_{1}, \cdots\right.$, $\left.X_{n}\right)+X_{0}^{m-2} G_{2}\left(X_{1}, \cdots, X_{n}\right)+\cdots+G_{m}\left(X_{1}, \cdots, X_{n}\right)$ where $m=\partial F_{1}$ and $i=\partial G_{i}$. Reducing modulo a we get $x_{0}^{m-1} g_{1} \in \mathfrak{p}^{2}$; but $x_{0}^{m-1} \notin \mathfrak{p}$ and $\mathfrak{p}^{2}$ is primary, hence $g_{1} \in \mathfrak{p}^{2}$ i.e. $G_{1} \in\left(X_{1}, \cdots, X_{n}\right)^{2}+\mathfrak{a}$ which implies $G_{1} \in \mathfrak{a}$. By repeating this argument we get $G_{1}, \cdots, G_{m} \in \mathfrak{a}$, therefore $\mathfrak{a}=$ $\left(G_{1}, \cdots, G_{m}, F_{2}, \cdots, F_{r}\right)$; the same for $F_{2}, \cdots, F_{r}$ and we are done.

COROLlaRY 3.2. With the same assumptions as above, if $P$ is regular the following conditions are equivalent:

(a) $\mathfrak{p}^{2}$ is primary.

(b) $\mathfrak{p}^{n}$ is primary for every $n$.

(c) $V$ is a linear space (i.e. a is generated by linear forms).

Proof. It follows from Theorem 3.1 that (c) and (b) are equivalent 
after remarking that a cone is a linear space if a point of its vertex is nonsingular for the cone. The equivalence of (a) and (b) follows from Theorem 2.2.

TheOREM 3.3. Let $X, Y$ be closed subschemes of $\boldsymbol{P}_{k}^{n}$, which are complete intersections in $\boldsymbol{P}_{k}^{n}$. Suppose that $Y$ is an irreducible, reduced, positive dimensional, normal subscheme of $X$ and $\operatorname{Sing}(X) \cap$ $Y \subseteq$ Sing $(Y)$ (where "Sing" stands for "singular locus of"). If $\mathfrak{p}$ denotes the prime ideal corresponding to $Y$ in the projective coordinate ring of $X$, then:

(a) $\mathfrak{p}^{2}$ is primary.

(b) If $\operatorname{dim} Y \geqq \operatorname{codim} X$, then $\mathfrak{p}^{n}$ is primary for every $n$.

Proof. If we denote by $\mathfrak{n}$ the ideal associated with $\operatorname{Sing}(Y)$, we get the proof as a strightforward consequence of Theorem 2.3.

COROLlary 3.4. With the same hypotheses of Theorem 3.3, if $X$ is a hypersurface, $\mathfrak{p}^{n}$ is primary for all $n$.

Now we shall try to justify the hypotheses of the previous theorems with same examples.

Example 1. In Theorem 3.1 and Corollary 3.2 the condition " $P$ rational" is essential. Let

$$
B=R\left[x_{0}, x_{1}, x_{2}\right] /\left(X_{0}^{2}+2 X_{1}^{2}-2 X_{1} X_{2}+X_{2}^{2}\right),
$$

$\mathfrak{p}=\left(x_{1}-x_{2}\right)$; we have $B / \mathfrak{p} \cong \boldsymbol{R}\left[X_{0}, X_{1}\right] /\left(X_{0}^{2}+X_{1}^{2}\right)$, hence $P$ is a nonrational closed point on $V$. By strightforward computation $V$ is a regular conic and $\mathfrak{p}^{n}$ is primary for all $n$ since $\mathfrak{p}$ is generated by a regular element of $B$, but $V$ is obviously not a linear space.

ExAMPLE 2. In Corollary 3.2 the condition " $P$ regular" is essential. Let $B=k\left[x_{0}, x_{1}, x_{2}\right] \cong k\left[X_{0}, X_{1}, X_{2}\right] /\left(X_{0} X_{1}^{2}-X_{2}^{3}\right), \mathfrak{p}=\left(x_{1}, x_{2}\right)$; it is clear that $\mathfrak{p}^{2}$ is primary.

REMARK. In Theorem 3.3 the condition " $\operatorname{dim} Y>0$ " is essential because if $\operatorname{dim} Y=0$ we have the counterexamples given by Theorem 3.1.

EXAMPLE 3. Let

$$
B=k\left[x_{0}, \cdots, x_{4}\right] /\left(X_{0} X_{2}-X_{3} X_{4}, X_{1} X_{2}-X_{3}^{2}, X_{0} X_{3}-X_{1} X_{4}\right),
$$

$\mathfrak{p}=\left(x_{2}, x_{3}, x_{4}\right)$. In this case the hypotheses of Theorem 3.3 are full- 
filled, save " $X$ complete intersection", and $\mathfrak{p}^{2}$ is not primary.

ExAmple 4. (see [2]). Let $X=\boldsymbol{P}_{k}^{5}, \mathfrak{p}$ the prime ideal defining the Veronese surface i.e. the prime ideal generated by the 2 by 2 minors of the matrix $M=\left(\begin{array}{lll}X_{0} & X_{1} & X_{2} \\ X_{1} & X_{3} & X_{4} \\ X_{2} & X_{4} & X_{5}\end{array}\right)$. In this case the hypotheses of Theorem 3.3 are fullfilled, save " $Y$ complete intersection", and $\mathfrak{p}^{2}$ is not primary. In fact if $d=\operatorname{det} M, d \notin \mathfrak{p}_{2}$ (for $\left.i=0, \cdots, 5\right)$.

EXAMPLE 5. Let $B=k\left[x_{0}, x_{1}, x_{2}, x_{3}\right] \cong k\left[X_{0}, X_{1}, X_{2}, X_{3}\left[/\left(X_{1} X_{2}-X_{3}^{2}\right)\right.\right.$, $\mathfrak{p}=\left(x_{2}, x_{3}\right)$. In this case the hypotheses of Theorem 3.3 are fullfilled, save "Sing $(X) \cap Y \subseteq \operatorname{Sing}(Y)$ ", and $\mathfrak{p}^{2}$ is not primary.

EXAmple 6. Let

$$
B=k\left[x_{0}, \cdots, x_{7}\right] /\left(X_{0} X_{2}+X_{1} X_{3}+X_{4}^{2}, X_{0} X_{5}+X_{1} X_{6}+X_{7}^{2}\right),
$$

$\mathfrak{p}=\left(x_{2}, \cdots, x_{7}\right)$. In this case the hypotheses of Theorem 3.3 are fullfilled hence $\mathfrak{p}^{2}$ is primary, but $1=\operatorname{dim} Y<\operatorname{codim} X=2$ and we are going to prove that $\mathfrak{p}^{3}$ is not primary. In fact if we call $f_{1}=$ $X_{0} X_{2}+X_{1} X_{3}+X_{4}^{2}, f_{2}=X_{0} X_{5}+X_{1} X_{6}+X_{7}^{2}$, from the identity

$$
X_{5} f_{1}-X_{2} f_{2}=X_{1}\left(X_{3} X_{5}-X_{2} X_{6}\right)+X_{5} X_{4}^{2}-X_{2} X_{7}^{2}
$$

we get $x_{1}\left(x_{3} x_{5}-x_{2} x_{6}\right)=x_{2} x_{7}^{2}=x_{5} x_{4}^{2} \in \mathfrak{p}^{3}$ with $x_{3} x_{5}-x_{2} x_{6} \in \mathfrak{p}^{3}$ and $x_{1} \notin \mathfrak{p}$.

\section{REFERENCES}

1. M. Teresa Bonardi, Alcune estensioni di un teorema di G. Z. Giambelli e R. Torelli ed applicazioni, Pubblicazioni dell' Istituto Matematico dell' Università di Genova, 1967.

2. - Sopra le potenze di un ideale primo di un anello di serie formali o convergenti, Le Matematiche, vol. XXIII, 1968.

3. Melvin Hochster, Criteria for equality of ordinary and symbolic powers of primes, Math. Zei., 133 (1973).

4. Irving Kaplansky, Commutative Rings, Allyn and Bacon, Boston, 1970.

5. David Rees, The grade of an ideal of module, Proc. Cambridge Phil. Soc., 53 (1957).

6. Oscar Zariaki and Pierre Samuel, Commutative Algebra, Vol. II, D. Van Nostrand, 1959 .

Received July 22, 1975 and in revised form December 9, 1975. This work was supported by C. N. R. (Consiglio Nazionale delle Ricerche).

Istituto Matematico Dell' Università di Genova

Via L. B. Alberti 4, Genova (Italia). 


\title{
PACIFIC JOURNAL OF MATHEMATICS
}

\author{
EDITORS
}

\author{
RICHARD ARENS (Managing Editor) \\ University of California \\ Los Angeles, California 90024
}

\author{
R. A. Beaumont \\ University of Washington \\ Seattle, Washington 98105
}

J. DugundjI

Department of Mathematics

University of Southern California

Los Angeles, California 90007

D. Gilbarg and J. Milgram

Stanford University

Stanford, California 94305

\section{ASSOCIATE EDITORS}
E. F. BECKENBACH
B. H. NEUMANN
F. WOLF
K. Yoshida

\section{SUPPORTING INSTITUTIONS}

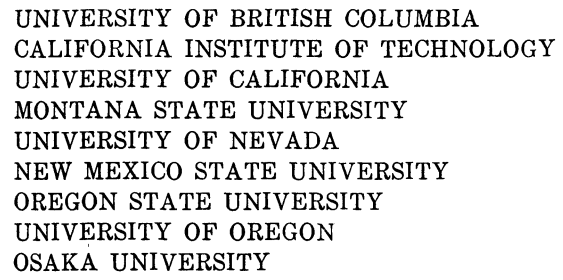

UNIVERSITY OF BRITISH COLUMBIA CALIFORNIA INSTITUTE OF TECHNOLOGY UNIVERSITY OF CALIFORNIA MONTANA STATE UNIVERSITY UNIVERSITY OF NEVADA NEW MEXICO STATE UNIVERSITY OREGON STATE UNIVERSITY UNIVERSITY OF OREGON OSAKA UNIVERSITY

\author{
UNIVERSITY OF SOUTHERN CALIFORNIA \\ STANFORD UNIVERSITY \\ UNIVERSITY OF HAWAII \\ UNIVERSITY OF TOKYO \\ UNIVERSITY OF UTAH \\ WASHINGTON STATE UNIVERSITY \\ UNIVERSITY OF WASHINGTON

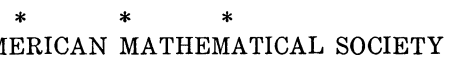

The Supporting Institutions listed above contribute to the cost of publication of this Journal, but they are not owners or publishers and have no responsibility for its content or policies.

Mathematical papers intended for publication in the Pacific Journal of Mathematics should be in typed form or offset-reproduced, (not dittoed), double spaced with large margins. Please do not use built up fractions in the text of your manuscript. You may however, use them in the displayed equations. Underline Greek letters in red, German in green, and script in blue. The first paragraph or two must be capable of being used separately as a synopsis of the entire paper. Items of the bibliography should not be cited there unless absolutely necessary, in which case they must be identified by author and Journal, rather than by item number. Manuscripts, in triplicate, may be sent to any one of the editors. Please classify according to the scheme of Math. Reviews, Index to Vol. 39. All other communications should be addressed to the managing editor, or Elaine Barth, University of California, Los Angeles, California, 90024.

The Pacific Journal of Mathematics expects the author's institution to pay page charges, and reserves the right to delay publication for nonpayment of charges in case of financial emergency.

100 reprints are provided free for each article, only if page charges have been substantially paid. Additional copies may be obtained at cost in multiples of 50 .

The Pacific Journal of Mathematics is issued monthly as of January 1966. Regular subscription rate: $\$ 72.00$ a year (6 Vols., 12 issues). Special rate: $\$ 36.00$ a year to individual members of supporting institutions.

Subscriptions, orders for back numbers, and changes of address should be sent to Pacific Journal of Mathematics, 103 Highland Boulevard, Berkeley, California, 94708.

PUBLISHED BY PACIFIC JOURNAL OF MATHEMATICS, A NON-PROFIT CORPORATION

Printed at Kokusai Bunken Insatsusha (International Academic Printing Co., Ltd.), 8-8, 3-chome, Takadanobaba, Shinjuku-ku, Tokyo 160, Japan.

Copyright (C) 1975 by Pacific Journal of Mathematics Manufactured and first issued in Japan 


\section{Pacific Journal of Mathematics}

\section{Vol. 63, No. 2 \\ April, 1976}

Joseph Anthony Ball and Arthur R. Lubin, On a class of contractive perturbations

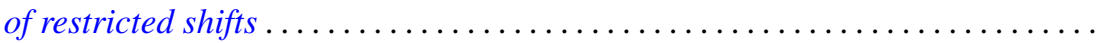

Joseph Becker and William C. Brown, On extending higher derivations generated

by cup products to the integral closure .......................

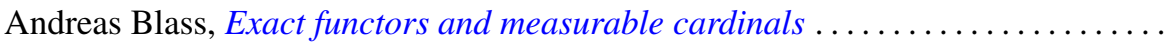

Joseph Eugene Collison, A variance property for arithmetic functions . . . . . . . . . .

Craig McCormack Cordes, Quadratic forms over nonformally real fields with a

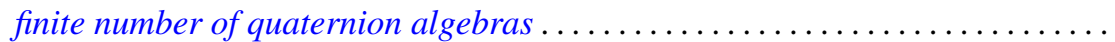

Freddy Delbaen, Weakly compact sets in $H^{1} \ldots \ldots \ldots \ldots \ldots \ldots \ldots \ldots \ldots$

G. D. Dikshit, Absolute Nörlund summability factors for Fourier series ..........

Edward Richard Fadell, Nielsen numbers as a homotopy type invariant. . ........

Josip Globevnik, Analytic extensions of vector-valued functions . . . . . . . . . . . .

Robert Gold, Genera in normal extensions . . . . . . . . . . . . . . . . . . . 389

Solomon Wolf Golomb, Formulas for the next prime

Robert L. Griess, Jr., The splitting of extensions of $S L(3,3)$ by the vector space

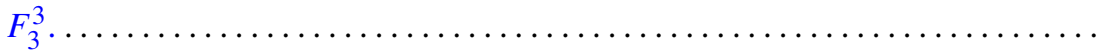

Thomas Alan Keagy, Matrix transformations and absolute summability .........

Kazuo Kishi, Analytic maps of the open unit disk onto a Gleason part.

Kwangil Koh, Jiang Luh and Mohan S. Putcha, On the associativity and commutativity of algebras over commutative rings . ..... . .

James C. Lillo, Asymptotic behavior of solutions of retarded differential difference equations.

John Alan MacBain, Local and global bifurcation from normal eigenvalues ..

Anna Maria Mantero, Sets of uniqueness and multiplicity for $L^{p}$

J. F. McClendon, Embedding metric families

L. Robbiano and Giuseppe Valla, Primary powers of a prime ideal .

Wolfgang Ruess, Generalized inductive limit topologies and barrelledness

properties.

Judith D. Sally, Bounds for numbers of generators of Cohen-Macaulay ideals

Helga Schirmer, Mappings of polyhedra with prescribed fixed points and fixed point indices.

Cho Wei Sit, Quotients of complete multipartite graphs

S. Sznajder and Zbigniew Zielezny, Solvability of convolution equations in $\mathscr{K}_{p}^{\prime}$,

$p>1$.

Mitchell Herbert Taibleson, The existence of natural field structures for finite

dimensional vector spaces over local fields

William Yslas Vélez, A characterization of completely regular fields

P. S. Venkatesan, On right unipotent semigroups ..............

Kenneth S. Williams, A rational octic reciprocity law ............

Robert Ross Wilson, Lattice orderings on the real field .......... 